\title{
ON THE LIFTING PROPERTY ${ }^{1}$
}

WILLIAM F. DONOGHUE, JR.

In the present note we give a simple proof of a result of von Neumann [3] using only the elementary theory of Banach algebras.

Theorem. In the space $L^{\infty}(R)$ of (equivalence classes of) bounded Lebesgue measureable function on the real axis, it is possible to choose representative functions $f^{*}(x)$ in each equivalence class $f$ in such a way that for any elements $f$ and $g$ of the space and any scalars $\alpha$ and $\beta$

$$
(\alpha f+\beta g)^{*}(x)=\alpha f^{*}(x)+\beta g^{*}(x)
$$

and

$$
(f g)^{*}(x)=f^{*}(x) g^{*}(x)
$$

Such determinations of representatives have been studied by $\mathrm{A}$. and C. Ionescu Tulcea [2] who call them liftings of $L^{\infty}(R)$. The theorem of von Neumann has been extended to general finite measure spaces by D. Maharam [1].

Since $L^{\infty}(R)$ is a commutative Banach algebra, it is clear that a lifting assigns to every real $x$ a multiplicative linear functional, viz. $F_{x}(f)=f^{*}(x)$. The null space of this functional is necessarily a maximal ideal, and since a neighborhood of the unit element consists exclusively of invertible elements, that ideal is not dense, and is therefore closed. Thus the multiplicative linear functional is continuous, and the lifting can be written

$$
f^{*}(x)=\hat{f}\left(M_{x}\right)
$$

where $\hat{f}$ is the Fourier-Gelfand transform of $f$ and $M_{x}$ the maximal ideal corresponding to $x$. On the other hand, if a mapping $x \rightarrow M_{x}$ of the real axis into the space of maximal ideals is given, then (1) describes a lifting if and only if for every $f$ the function $f^{*}(x)$ belongs to the equivalence class $f$. We will show the existence of a mapping having this property.

Let $e$ be an arbitrary idempotent in $L^{\infty}(R)$, i.e. an element for which $e^{2}=e$. We assign a support $E$ to $e$ as follows: the equivalence

Received by the editors June 22, 1964.

1 The work presented in this paper was done while the author was at the Courant Institute of Mathematical Sciences, New York University, supported by a Ford Foundation grant. 
class $e$ contains a characteristic function of a measurable set $S$, and we let $E$ be the set of points of density of the set $S$. This definition of the support $E$ is independent of the particular choice of the characteristic function in $e$; in fact, $E$ could also have been defined as the set of points $x$ where the function $\int_{0}^{x} e(t) d t$ has the derivative $+1, e(t)$ being any function in the equivalence class $e$. It is important to remark that if $e_{1}$ and $e_{2}$ are two idempotents, with supports $E_{1}$ and $E_{2}$ respectively, then any point $x$ in $E_{1} \cap E_{2}$ is in the support of the product $e_{1} e_{2}$, which therefore is not zero.

Given a real $x$, consider $\mathcal{F}_{x}$, the family of all idempotents for which $x$ is in the support; in view of the previous remark $\mathcal{F}_{x}$ is closed under multiplication. Passing to the Fourier-Gelfand transforms, we obtain a family of closed subsets of the compact space of maximal ideals, viz. the family of sets $\hat{e}(M)=1$ as $e$ runs through $\mathcal{F}_{x}$. This family of sets has the finite intersection property, hence there exists at least one point $M_{x}$ such that $\hat{e}\left(M_{x}\right)=1$ for all $e$ in $F_{x}$. If to each $x$ we assign such an $M_{x}$ we find for every idempotent $e$ that $e^{*}(x)=\hat{e}\left(M_{x}\right)$ is a characteristic function of a set containing the support $E$ of $e$. Similarly $(1-e)^{*}(x)=1-e^{*}(x)$ is the characteristic function of the complementary set which contains the support of $1-e$. Since the two supports cover the axis, save for a set of measure 0 , it follows that $e^{*}(x)$ is in the equivalence class $e$.

If $f$ is a finite linear combination of idempotents we have

$$
f^{*}(x)=\left(\sum a_{k} e_{k}\right)^{*}(x)=\sum a_{k} e_{k}^{*}(x)
$$

which is evidently a bounded measurable function in the equivalence class of $f$. Since the class of such functions is dense in $L^{\infty}(R)$, it follows that (1) defines a lifting of $L^{\infty}(R)$.

\section{REFERENCES}

1. D. Maharam, On a theorem of von Neumann, Proc. Amer. Math. Soc. 9 (1958), 987-994.

2. A. Ionescu Tulcea and C. Ionescu Tulcea, On the lifting property. I, J. Math. Anal. Appl. 3 (1961), 537-546.

3. J. von Neumann, Algebraische Repräsentanten der Funktionen bis auf eine Menge von Masse Null, J. Reine Angew. Math. 165 (1931), 109-115.

New York University 